\title{
Restablecimiento del Derecho a la Educación: Identidad Narrativa de Mujeres Mayores
}

\author{
Restoring the Right to Education: Narrative Identity of Old \\ Women
}

\author{
Karina Moreno Díaz ${ }^{1 *}$ \\ Rodolfo Soto González ${ }^{2}$ \\ ${ }^{1}$ Fundación Belén Educa \\ ${ }^{2}$ Centro de Formación Técnica ENAC
}

\begin{abstract}
Una proporción significativa de mujeres mayores no accedieron a la educación formal, viéndose vulnerado su derecho a la educación. En este contexto, la educación popular se presenta como una alternativa de alfabetización para este grupo. Esta investigación tiene por objetivo comprender la identidad narrativa de mujeres mayores que han participado en el plan popular de alfabetización Contigo Aprendo. Dado el carácter del objeto de estudio, se optó por utilizar un enfoque cualitativo de investigación. Así, las técnicas de producción incluyeron entrevistas narrativas a 10 mujeres mayores y un focus group, siendo la información analizada en base a la teoría fundamentada. Los resultados muestran que la vulneración del derecho a la educación de las mujeres durante la infancia y la adultez fue ocasionada por una postergación por labores de cuidado y la decisión de personas del contexto familiar. En la vejez se presenta una liberación de tales labores y la motivación por aprender y empoderarse puede concretarse con la participación en el plan de alfabetización. Estos resultados se discuten considerando, en primer término, la manera en que las restricciones a la educación formal estuvieron moldeadas por estereotipos de género $y$, en segundo término, el impacto de la educación popular para restituir el derecho a la educación.
\end{abstract}

Descriptores: Derecho a la educación, Identidad, Alfabetización, Vejez, Educación de la mujer.

A significant proportion of older women did not access to formal education, thus violating their right to education. In this context, the popular education is presented as a literacy alternative for this group. This research aims to understand the narrative identity of older women who participated in the popular literacy plan Contigo Aprendo (translated as I learn with you). Given the nature of the object of study, we chose to use a qualitative research approach. Thus, production techniques included narrative interviews to 10 older women and a focus-group interview, the information was analyzed using the Theory Grounded method. The results show that the infringement to the right to education of women during childhood and adulthood was driven from its postponement from care labor and decision from other persons in the family. At old ages, freedom from those labors arises, and motivation to learn and empower themselves can result in the participation on the literacy program. These results are discussed, in first place, considering how restrictions on formal education were shaped by gender stereotypes and, secondly, the impact of popular education on the restitution of the right to education.

Keywords: Right to education, Identity, Literacy, Old age, Women education.

*Contacto: karina.moreno@usach.cl

ISSN: 0718-7378

www.rinace.net/rlei/ $\begin{array}{ll}\text { Recibido: } & 01 / 07 / 2017 \\ 1^{a} \text { Evaluación: } & 12 / 09 / 2017 \\ \text { Aceptado: } & 02 / 10 / 2017\end{array}$ 


\section{Problema de investigación}

La última Encuesta de Caracterización Socioeconómica Nacional -CASEN- (Ministerio de Desarrollo Social, 2015) indica que en Chile existen 3.076.000 de adultos mayores, de los cuales el $57.3 \%$ son mujeres, mostrando un índice de envejecimiento en aumento y una feminización de la tercera edad (Comunidad Mujer, 2016a). Según la Organización Mundial de la Salud, Chile es el país de América Latina con mayor expectativa de vida, correspondiente a 80.5 años en promedio. Específicamente, la expectativa de vida para las mujeres es de 83.4 años y 77.4 años para los hombres (World Health Organization, 2016).

Este aumento en la expectativa de vida plantea importantes desafíos a nivel social en diferentes áreas como la salud, las pensiones y la educación (Mogollón, 2012), especialmente en el caso de las mujeres. En particular, las mujeres mayores tienen en promedio solo 7.7 años de estudios, a diferencia de los 12 años de escolaridad que alcanza el resto de la población (Ministerio de Desarrollo Social, 2015). Esta escasa tasa de escolarización de las mujeres mayores se debe principalmente a su exigua participación en el campo educativo durante la primera mitad del siglo Xx (Cannobbio y Jeri, 2008). Según Valdés (1987), esta situación se deriva de los "espacios culturales" asociados al género femenino predominante en la época, donde el mundo privado era el lugar legítimo para la mujer, desempeñando roles como "madre", "esposa" y "dueña de casa" (Eltit, 1994). Con esto, su derecho a la educación se vio vulnerado al no acceder a las mismas oportunidades educativas que los hombres, situación que tiene consecuencias hasta hoy (Cannobbio y Jeri, 2008) no solo en Chile sino también en otros países de América Latina. Es así como es una de las regiones con mayor número de adultos analfabetos y que no han concluido sus estudios primarios (Comboni y Juárez, 2005)

De este modo, se cuenta actualmente con un grupo humano cuyo ejercicio del derecho a la educación fue restringido por las condiciones sociales de la época en que debieron acceder a la educación formal. Sin embargo, gracias al aumento en la expectativa de vida de las mujeres, a la explicitación del potencial de los adultos mayores para insertarse en la educación de manera permanente (Mogollón, 2012) y al actual paradigma del aprendizaje a lo largo de toda la vida, se abre una importante oportunidad para restablecer el derecho a la educación de las mujeres mayores. Una alternativa para este restablecimiento es la alfabetización, ya que es inherente al derecho a la educación y es la base más importante sobre la cual se construye el aprendizaje tanto de jóvenes y adultos (UNESCO, 2009).

Una alternativa que ha surgido con fuerza en el último tiempo para alfabetizar y trabajar con adultos mayores es la educación popular propuesta por Paulo Freire, la cual destaca por su capacidad de adaptarse a las características, necesidades y exigencias de este grupo (Esquivel, 1999) y por considerar a la alfabetización como una forma de empoderamiento personal (Freire, 2004). Es precisamente este enfoque el que fundamenta el Plan Contigo Aprendo, alternativa con la cual el Ministerio de Educación busca entregar una oportunidad de aprendizaje para quienes requieran aprender a leer, escribir y desarrollar el pensamiento matemático y, con ello, puedan certificar $4^{\circ}$ año básico (MINEDUC, 2017).

Según la UNESCO (2009), este tipo de programas con foco en la alfabetización no solo permite a las personas adquirir habilidades de lectoescritura, sino que a su vez impacta 
en su autonomía e identidad personal. Este impacto se fundamenta en que la alfabetización, desde la mirada de Freire, permite a las personas darse cuenta de su realidad sociopolítica e iniciar un proceso de autoformación, creación y re-creación personal. De este modo, la alfabetización genera en las personas conversaciones y deliberaciones en torno a sus vivencias en sus propios contextos y en cómo estas influyen en su identidad (Freire, 2004).

Por tanto, la identidad personal es transformada con la participación en instancias comunitarias y educativas (Wenger, 2002). En este contexto y como la identidad se plasma en construcciones narrativas que los sujetos relatan en primera persona (Bolívar, 2002), para comprender la influencia que tiene en la persona la inclusión y/o exclusión en procesos formativos es necesario rescatar sus narraciones. Esta labor permitiría entender el grado de cumplimiento del derecho a la educación en grupos específicos, como las mujeres mayores, especialmente si se concibe este derecho desde el ejercicio concreto de las personas y no como un lineamiento que se encuentra cosificado en un documento legal (Liebel, 2013). Por otro lado, la cualidad de las narraciones para capturar la dimensión temporal (Bruner, 2004) también facilita la comprensión de la alfabetización como un proceso que dura toda la vida (UNESCO, 2009).

Por su parte, es importante considerar que la alfabetización va más allá de aprender a leer y escribir y que, además, es parte consustancial del derecho a la educación. Por ende, el Plan Contigo Aprendo se transforma en un espacio que tiene el potencial de restituir este derecho por medio de la alfabetización. Asimismo, y considerando que uno de los principales grupos afectados por la vulneración del derecho a la educación son las mujeres y que son ellas las principales participantes del plan, con una presencia del $75.3 \%$, se torna relevante estudiar sus experiencias de vida, en especial de las que componen el tramo etario de 50 años en adelante, ya que su participación en el plan corresponde al 31.9\% (Escobar, Berlien y Ostoic, 2016). De este modo, es menester capturar el relato de estas experiencias en el contexto de su vida e identidad para vislumbrar cómo tuvo lugar la vulneración y la eventual restitución del derecho a la educación. En suma, se establece como objetivo de la presente investigación comprender la identidad narrativa de mujeres mayores que han participado en el plan popular de alfabetización Contigo Aprendo.

\section{Antecedentes teóricos e históricos}

\subsection{Mujeres y educación}

Según Eltit (1994), las mujeres han recorrido un extenso camino para acceder plenamente a la educación, transitando lentamente desde el espacio privado hasta ser parte de las distintas esferas sociales. Es así como en 1812, a pocos años del nacimiento de la República, solo el $10 \%$ de la población femenina sabía leer y el $8 \%$ escribir. Posteriormente, en el gobierno de Manuel Montt se realizaron importantes avances en las políticas educacionales, destacando el carácter gratuito de la educación para ambos sexos y la construcción de una escuela cada 2.000 habitantes. De este modo, en 1882 el $32 \%$ de las mujeres ya estaban alfabetizadas. Otro importante hito lo representa el denominado Decreto Amunátegui que permitía que las mujeres accedieran a la educación superior. 
A pesar de que estos avances facilitaron la incorporación de las mujeres a la educación, esta inserción ha estado marcada por la desigualdad debido principalmente a los denominados espacios culturales del género femenino. Estos espacios refieren a las áreas en que la presencia de la mujer es legítima y requerida para el funcionamiento de la sociedad (Valdés, 1987). Estos espacios aluden principalmente al mundo privado, enfocado a los roles de madre, esposa y dueña de casa (Eltit, 1994; Valdés, 1987). Debido a lo anterior, se plantea que la mujer ha estado triplemente desventajada en relación al hombre, considerando su inserción al mundo laboral, educativo y público (Verba, 1978). Esta situación generó la inhibición de la mujer frente a las actividades extra hogareñas (Didier, 1990), de modo que la decisión de participar en alguna organización social debía pasar por la aprobación de la pareja (Valdés, 1987).

Por consiguiente, la participación de la mujer en la esfera educativa durante la primera mitad del siglo Xx fue escasa (Cannobbio y Jeri, 2008). Esta situación fue cambiando con el paso del tiempo en virtud de la implementación de nuevas políticas educacionales y cambios sociales en torno al rol de la mujer, de modo que actualmente no existen brechas significativas en el acceso de las mujeres a nivel parvulario, básico, medio ni superior de educación (Comunidad Mujer, 2016b). Sin embargo, aún quedan consecuencias del periodo en el cual el rol de las mujeres se circunscribía exclusivamente al espacio privado y donde la educación básica no estaba masificada ni garantizada para ellas (Cannobbio y Jeri, 2008).

Hoy por hoy, si se considera el total de adultos mayores que no saben leer ni escribir correspondiente al $8.3 \%$, las mujeres superan a los hombres. Específicamente, de este porcentaje el $58.7 \%$ corresponden a mujeres y un $41.3 \%$ a hombres ${ }^{1}$. Asimismo, los datos indican que el analfabetismo se acrecienta a medida que aumenta la edad, manteniendo las mujeres la tasa más alta. En cuanto al acceso efectivo a la educación formal, las mujeres mayores de 60 años tienen en promedio solo 7.7 años de estudios en contraposición a los hombres que poseen 8.4 años de estudios formales en las mismas condiciones (Ministerio de Desarrollo Social , 2015). Se agrega a las cifras anteriores que el $8 \%$ de los adultos mayores no tuvo acceso al sistema de educación formal, porcentaje del cual $61.9 \%$ corresponden a mujeres y solo el $38.1 \%$ a hombres 2 .

\subsection{Adulto mayor y educación popular}

La educación es un derecho que no se circunscribe a una etapa de la vida, ya que el aprendizaje va "de la cuna a la tumba". La afirmación anterior fundamenta el paradigma denominado aprendizaje a lo largo de toda la vida, el cual constituye un marco filosófico y conceptual basado en valores inclusivos, emancipatorios, humanísticos y democráticos, siendo esencial para lograr una sociedad basada en el conocimiento y la justicia. Un componente central de este paradigma es la educación para adultos, la cual atiende las necesidades de aprendizaje de los jóvenes, adultos y adultos mayores y los dota de conocimientos, capacidades, habilidades, competencias y valores para ejercer y promover sus derechos (UNESCO, 2009).

Basándose en lo anterior, hacia finales de 1990 comenzaron a surgir diferentes propuestas políticas y educativas referidas a la educación de adultos, las cuales

${ }^{1}$ Cifras elaboradas por los autores a partir de los datos de la CASEN 2015.

${ }^{2}$ Cifras elaboradas por los autores a partir de los datos de la CASEN 2015. 
comprendían desde la alfabetización hasta la educación superior (UNESCO, 2010). Sin embargo, estas iniciativas escasamente apuntaban a los adultos mayores debido principalmente a los estereotipos sobre este grupo humano (García, 2007) y a su supuesta condición de vulnerabilidad física, psicológica y de su contexto social. Esta concepción negativa acerca de la vejez ha ido cambiando gracias a investigaciones gerontológicas en distintos ámbitos, las cuales muestran que los adultos mayores poseen el potencial para insertarse en la educación de forma permanente (Mogollón, 2012).

A pesar de este cambio en la visión de la vejez y su relación con la educación, el enfoque educativo tradicional ha estado en deuda con las personas mayores pues no cuenta con los espacios para que puedan transmitir sus experiencias, extender su formación personal y así continuar aprendiendo y enseñando (García, 2007). En respuesta, surgen nuevas estructuras acordes al aprendizaje a lo largo de la vida que consideran a los adultos mayores (García, 2007), dentro de las que destaca la educación popular de Paulo Freire, la cual está orientada a concientizar a los grupos oprimidos (Freire, 1985) e influyó en las renovaciones pedagógicas de América Latina, África y Europa (Torres, 2007). La educación popular tiene como principales características la transformación del individuo por medio de la crítica y la concientización, junto con la utilización de métodos principalmente orales (Freire, 2004).

Dada las características de la educación popular y su versatilidad para desarrollarse en múltiples formas, es particularmente útil para trabajar con adultos mayores, ya que se puede adaptar fácilmente a sus necesidades, características y exigencias. De este modo, desde la educación popular los adultos mayores son repensados como sujetos políticos y protagonistas de un proyecto histórico-social y su foco está en la recuperación de un conjunto de elementos históricos. En consecuencia, se busca problematizar el presente nacional, comunal y familiar en base a su vida, al recorrido del país y a la serie de acontecimientos políticos, económicos y culturales (Esquivel, 1999).

Tomando en consideración el enfoque de educación de adultos propuesto por la educación popular, el Ministerio de Educación desarrolla el plan Contigo Aprendo con el objetivo que adultos desde los 15 años puedan aprender a leer y escribir, desarrollen su pensamiento matemático y, en consecuencia, puedan certificar $4^{\circ}$ año básico (MINEDUC, 2017). Uno de los principales grupos objetivos de este plan son los adultos mayores, ya que su implementación es parte de las políticas sociales dirigidas al adulto mayor en el área de la educación (Lampert-Grassi, 2014). Específicamente, el plan Contigo Aprendo rescata del enfoque popular la valorización y uso de las experiencias y saberes previos con que los adultos llegan a integrarse al plan. Asimismo, Contigo Aprendo indica considerar los elementos más significativos del contexto de las personas de modo de construir una relación entre lo que son, lo que viven, el lugar donde viven y lo que ahí aprende (Hernández, 2009).

\subsection{Narración e identidad}

Bruner (2004) postula que la mente se organiza bajo dos modalidades: narrativa y paradigmática. Esta última destaca el empleo de la categorización y la conceptualización. Por su parte, en la modalidad narrativa predominan las vicisitudes de las intenciones humanas. Los componentes de la narración incluyen el actor, la acción, la meta o intención, el escenario, el instrumento y el problema. Estos elementos se enmarcan en una secuencia, que globalmente configuran la trama de la narración. Los relatos se configuran en un "paisaje dual" compuesto por la acción y la consciencia. En el primero 
se encuentran la secuencia de acontecimientos y las acciones en el mundo y, paralelamente se expresa el segundo paisaje, en el cual se muestran las ideas e intenciones en la consciencia de los participantes (Bruner, 1990). Por tanto y a diferencia de la modalidad paradigmática, las narraciones admiten los sentimientos, las intenciones y el contexto de sus actores.

Estas características permiten que las narraciones formen presentaciones miméticas de la vida humana, sin ser una representación objetiva (Flick, 2007). En otras palabras, la narración es el medio más adecuado para elucidar la experiencia temporal, puesto que la narratividad la determina, articula y clarifica (Ricoeur, 1995). Mediante este proceso de construcción se va constituyendo a su personaje, es decir, a quién se le atribuyen las acciones e intenciones del relato. La identidad narrativa corresponde a la unidad narrativa de las experiencias durante el transcurso de la vida, dando acceso al quién a través de la historia (Arciero, 2005). La identidad implica el factor temporal y, por ende, las historias personales reflejan trayectorias de aprendizaje producto de la participación en diferentes contextos sociales (Wenger, 2002).

Las narrativas y sus técnicas de investigación asociadas facilitan la recuperación de los puntos de vista de las personas, rescatando tanto lo particular como la dimensión emotiva de la experiencia humana. Estos atributos de la narración la posicionan en un rol privilegiado para comprender desde "dentro" las vivencias de exclusión de las personas y su construcción (Susinos y Parrilla, 2008). Este proceso de construcción social de la exclusión consiste en la denegación para ciertos grupos de personas al acceso y disfrute de determinados derechos de ciudadanía (Tezanos, 2001), como el derecho a la educación.

\section{Método}

Ajustándose al problema de investigación planteado, se consideró necesaria la elección de estrategias metodológicas propias del enfoque cualitativo. En efecto, la aplicación de este enfoque permite el acercamiento a las perspectivas en contexto y el estudio de significados narrativos (Flick, 2007). Estas características del enfoque cualitativo permiten profundizar en la identidad narrativa como objeto de estudio. En el contexto de esta investigación, las herramientas cualitativas permiten asistir la exploración de la identidad narrativa de mujeres mayores, facilitando la construcción de narraciones articuladas sobre sus experiencias vitales contextualizadas en la inclusión y/o exclusión en espacios educativos y su posterior análisis a la luz del derecho a la educación.

\subsection{Participantes}

Las participantes de este estudio asistieron al plan de alfabetización "Contigo Aprendo", implementado en las dependencias de dos establecimientos educacionales de la Región Metropolitana. Se emplearon tres criterios de selección de las participantes: a) su género correspondiera al femenino, b) tuvieran como mínimo 60 años cumplidos y c) debían haber participado al menos durante un año en este plan a objeto de disponer de un periodo significativo de experiencias. De esta manera, participaron en esta investigación 10 mujeres mayores cuyas edades se ubicaban entre los 62 a 76 años. El consentimiento informado se obtuvo en presencia de un testigo independiente al equipo de investigadores. 


\subsection{Procedimiento de producción de información}

Con la finalidad de comprender la identidad narrativa de las participantes, se optó por la técnica de la entrevista narrativa. Esta técnica se basa en la solicitud al informante para que presente una historia del o las áreas de interés investigativo, obteniendo un relato coherente de los acontecimientos relevantes (Hermanns, 1995). Las áreas de interés en el contexto de esta investigación refieren principalmente a las etapas de la infancia, a la adultez y a la vejez, considerando su relación con la educación. Además, se incluye durante la vejez su participación en el plan de alfabetización "Contigo Aprendo". Esta técnica admite en su parte final preguntas de profundización e intervenciones concretas (Flick, 2007).

Si bien la entrevista narrativa fue la técnica principal de producción de información, también se realizó un grupo focal con cinco mujeres que asistían juntas al plan de alfabetización. Esta técnica fue aplicada para indagar en significados compartidos respecto tanto a la experiencia en el plan de alfabetización como en procesos vitales comunes.

\subsection{Procedimiento de análisis de información}

El procedimiento de análisis aplicado siguió las orientaciones de la Teoría Fundamentada (Strauss y Corbin, 2002). Concretamente, se utilizaron tres técnicas específicas de esta metodología de análisis: la codificación abierta, axial y de proceso. La primera técnica sirvió para generar categorías que reflejaron significados narrativos de los participantes. La codificación axial fue empleada para relacionar dichas categorías en ejes, los cuales expresan etapas vitales. Posteriormente, se hizo uso de la codificación de proceso para articular los significados narrativos de cada etapa vital en un curso temporal. La construcción de esta historia articulada permitiría mostrar la unidad característica de la identidad narrativa. Las dos técnicas de integración para caracterizar la identidad narrativa como unidad corresponden al argumento de la historia y al esquema, empleando paralelamente una representación verbal y gráfica del objeto de estudio. El argumento de la historia utiliza las categorías y los vínculos analizados para formar oraciones conectadas, de tal manera que se origina una historia que encaja con los datos (Strauss y Corbin, 2002).Cabe señalar que estos procedimientos analíticos fueron realizados mediante el programa Atlas.ti.

\section{Resultados}

En esta sección se muestran las categorías construidas en el proceso de codificación. Estas categorías se encuentran organizadas en cuatro apartados que representan las etapas vitales de las participantes y su participación en el plan: infancia, adultez, vejez y plan.

\subsection{Infancia}

\subsubsection{Figura parental negativa y/o restricciones socioeconómicas}

Las participantes indican que en su infancia las condiciones familiares suponían, en la mayor parte de los casos, un tipo de relación que producía sentimientos de descontento con al menos uno de sus padres o cuidadores. Por lo tanto, la relación con alguno de los padres es calificada negativamente e incluían formas de maltrato y abandono. 
Mi madre era muy mala, yo la encuentro mala porque ahora yo soy madre y yo no fui con mis hijos como ella fue conmigo... Mala porque por cualquier cosa me pegaba, me trataba mal, desgraciada para acá, desgraciada para allá.

El escenario familiar también estaba caracterizado por la presencia de limitaciones socioeconómicas. En particular, los padres o cuidadores no disponían de un nivel educativo básico y realizaban labores generalmente de carácter manual que les permitía acceder a escasos ingresos monetarios.

Entonces no podíamos venir al colegio. Mi mamá no tenía y mi papá era un obrero no más. Si usted sabe que antes no pagaban como ahora.

\subsubsection{Negación de participación en la escuela}

A todas las participantes de este estudio, en alguna medida, se les restringió su derecho a la educación durante la infancia. Generalmente, la decisión de negar la participación en el proceso de escolarización emanaba desde alguno de sus padres. También jugaba un rol importante las limitaciones socioeconómicas que presentaba el escenario familiar.

Yo fui muy poco al colegio porque mi papá era un hombre alcohólico, enfermo, éramos 6 hermanos, lo cual lo tuvieron que internar, entonces ese fue el motivo que estábamos un tiempo en el colegio y él los sacaba y mi mami tenía que salir con nosotros arrancando porque él hacía sus cosas.

\subsubsection{Dedicación a labores de cuidado, domésticas y/o del campo}

Las mujeres señalan que destinaron su tiempo durante la infancia a labores que no contemplaban su participación en programas formales de estudio. En esta línea, presentaron una dedicación focalizada en actividades de cuidado principalmente ligada a la crianza de sus hermanos y a tareas domésticas. Además, algunas participantes se ocupaban en esta etapa de labores relacionadas al campo.

\footnotetext{
ro no pude estudiar en su momento porque éramos tantos hermanos, que yo soy de las mayores de mis hermanas, somos 6 hermanas y entonces no podíamos ir al colegio porque yo tenía que cuidar a mis hermanos.

Tuve una niñez muy mala. Ayude a mi mamá a lavar a la gente más poderosa pongámosle, ropa, calcetas, de todo y de ahi ya fuimos creciendo.
}

\subsubsection{Rechazo de condiciones con intención de escapar}

Estas condiciones en la infancia fueron estimadas como negativas por las mujeres y generaban sufrimiento en el plano emocional. Como respuesta a estos sentimientos, las participantes iban adoptando una intención de escapar del contexto familiar primario.

Como yo era la mayor, yo quería salir de la casa, estaba aburrida con tanto.

\subsection{Adultez}

3.2.1. Autoconcepción desde género femenino con orientación tanto al apoyo como a labores de cuidado y domésticas

La concepción que las participantes tienen de sí mismas está caracterizada con una focalización en la ayuda hacia los demás. Además, esta orientación al apoyo social es experimentada especialmente con los hijos, es decir, desde el rol materno. Las tareas domésticas en el hogar también permean esta autoconcepción.

Me veo buena persona, si le puedo hacer un favor al que sea se lo voy a hacer con mi esposo, con mis hijos... se lo voy a hacer de todo corazón sin esperar recompensa, así 
me describo yo y a quien sea. Aunque sea de la calle también porque se merecen derechos también, que uno les tienda la mano.

Ser mujer no tendría que afectarle a uno, al contrario, debería sentirse orgullosa, ser madre y ser mujer, ser dueña de casa.

\subsubsection{Embarazo y casamiento como entrada a la adultez}

El relato de las participantes revela que el inicio a la adultez habitualmente estaba marcado por el embarazo y, posteriormente, con la concreción del matrimonio. Esta situación derivaba de la intención de escapar indicada previamente y, en algunas ocasiones, el matrimonio se producía con presión de los padres.

Mi mamá lavaba, lavaba todos los días con escobillita. To la veía y decía y si me
caso mejor, voy a salir de acá, de mis hermanos y de ahí, después que yo ya estaba
embarazada, no tenía idea mi madre me dijo que me tenía que casar con mi esposo
que tenía 28 años.

3.2.3. Matrimonio como experiencia negativa con negación de participación en proceso educativo

La información biográfica recopilada da cuenta que la experiencia vivida en el matrimonio presenta dimensiones que son connotadas afectivamente como negativas. En concreto, se imponen limitaciones desde el esposo para participar en otras esferas sociales más allá del hogar. El ámbito educativo se encuentra particularmente con fuertes restricciones.

\section{Bueno, yo allí ya empecé una edad de mamá y después me casé con el papá de mi hija y así nunca he estudiado, porque también fue machista. To siempre tuve la intención de estudiar, pero como era, yo vivía con lo que él me daba, nunca salí a la calle.}

\subsubsection{Consecuencias negativas y vergüenza por escasez de aprendizajes}

Las participantes señalan que la baja o nula participación en experiencias formativas provocaba distintos efectos negativos en sus vidas. La escasa alfabetización generaba barreras en la inserción laboral, en la realización de trámites e, incluso, en la implicación en escenarios recreativos. No obstante, la consecuencia más preponderante fue la experimentación de vergüenza e inseguridad.

\footnotetext{
Si a usted la hacían firmar, nunca firmábamos, firmábamos con el dedo, que vergüenza decía yo una vez, que vergüenza y de ahí hasta, no, ahora que vergüenza. Hay tanta gente que no sabe leer ni escribir como nosotros.
}

\subsubsection{Dedicación a labores de cuidado y/o trabajo por hijos}

Durante la adultez, las actividades realizadas por las mujeres estuvieron relacionadas fundamentalmente con el cuidado de los hijos. En otras palabras, las participantes ejecutaban en la rutina diaria labores de crianza y también ejercían actividades remuneras para sustentar a sus hijos.

To siempre decía, me voy a casar, me voy a casar para no trabajar, trabajé doble, porque empezaron a llegar los hijos y una vez que están hechos ya no se pueden deshacer y hay que tenerlos nomás.

\subsubsection{Postergación por labores de cuidado}

Dado que en la etapa de adultez las mujeres invirtieron su tiempo principalmente en tareas de cuidado de sus hijos, postergaron la realización de variados intereses personales. Por ejemplo, aunque algunas mujeres deseaban "irse” del hogar, optaron por mantenerse porque consideraban que sus hijos eran beneficiados. 
Tuve 2 veces por irme eso sí, pero miraba a mis hijos y ellos no tenían culpa. Allá me quedé y seguí con ellos luchando hasta que salí con ellos adelante.

\title{
3.2.7. Anhelo de participación en proceso de aprendizaje
}

Según el discurso de las mujeres, la intención de participar en algún proceso formativo que les permitiera adquirir habilidades vinculadas a la alfabetización estuvo constantemente presente durante la adultez y, en algunas, desde la infancia.

Obvio, yo siempre he soñado que voy a estudiar, pero nunca me dio la oportunidady ahora que la tuve no la suelto, ahora voy a seguir estudiando.

\subsection{Vejez}

\subsubsection{Satisfacción por libertad de labores de cuidado}

En términos generales, la vejez de las mujeres está marcada por el desprendimiento de responsabilidades de crianza. Esta reducción en exigencias asociadas al rol parental produce en ellas un significativo estado emocional de satisfacción. Esta mayor percepción de libertad es, desde su mirada, una condición alcanzada por su "sacrificio" durante su adultez.

\begin{abstract}
Así que esa ha sido mi vida dije que de primera cuando mis hijos estuvieron chicos hasta la edad en que terminó mi hija chica de estudiar que tenía 18 años, hasta ahí fue mi sufrimiento grande, toda la época de la niñez de ella y el marido que tenía que era un desastre. Después él se fue de al lado y mis hijos crecieron, ya mi vida se empezó a alivianar. En estos momentos si usted me pregunta yo le digo que soy la persona más feliz que puede haber, en todo sentido porque si yo quiero hago las cosas y si no quiero no las hago y a mí nadie me llama la atención.
\end{abstract}

Dios me está premiando por lo buena que fui poh, buena con mis hijos.

\subsubsection{Satisfacción por apoyo de hijos}

En el relato de las participantes se manifiesta, en distintos niveles de intensidad y regularidad, el apoyo de los hijos que experimentaron su dedicación en materia de crianza. Este suministro de ayuda también alimenta un mayor nivel de satisfacción en la tercera edad.

Ahora soy feliz porque no tengo problemas de nada, por nadien y al contrario ahora
ellas me ayudan a mí, están pendientes de mí, que no me falte nada, que cómo
amanezco, que, si tengo algo que comprar, la chica por ejemplo siempre me llama
todos los días mamita tenís remedio que comprar, no mi amor si los remedios que
tomó me los dan en el consultorio, mamita cuando tengaí algo que comprar y no
tengaí plata llámame no más y yo te mando una monea 3.3.3. Mayor dedicación a sí mismas

Las participantes explicitan que, a diferencia de su perspectiva en la adultez, no les preocupan mayormente las opiniones de las demás personas. En contraste, su atención está más concentrada en sí mismas, lo cual se reconoce como legítimo producto de su abnegación en la adultez.

Más feliz porque no hay problema, entonces uno lo único que hace es que se dedica a lo que tiene uno, a lo que tiene uno entonces nadie la crítica y la que la crítica, la crítica por detrás, pero uno no escucha.

\subsubsection{Motivación por incorporarse en proceso formativo}

La motivación indicada por las participantes en la adultez también se manifiesta en el relato que hacen de su vejez y actualidad. Esta motivación se revela como una necesidad 
de recibir enseñanza para, por ejemplo, escribir una carta, leer un libro, disponer una actividad para ocupar el tiempo libre o experimentar un sentimiento de superación.

No voy a parar hasta que aprenda a leer de corrido.

\subsubsection{Anhelo y satisfacción por mayor empoderamiento}

Las participantes perciben que disponen de mayor independencia en la vejez producto de la dedicación a sí mismas. Esta independencia se expresa con un mayor poder de decisión en su esfera personal, lo que a su vez genera un nivel superior de disfrute de las experiencias de vida.

He cambiado, soy más terca ahora, tengo más experiencia, ahora tiene que ser lo que yo diga y tiene que ser lo que yo diga.

\subsection{Participación en plan}

\subsubsection{Plan como oportunidad}

Como se describió anteriormente, tanto la intención de participar en una instancia de alfabetización como una percepción de mayor empoderamiento personal se manifestaban significativamente en la vejez. Estos elementos establecían condiciones favorables para que las participantes se inscribieran en el plan de alfabetización una vez identificado.

To siempre dije, cuando yo no tenga más problemas, cuando ya no trabaje, no tenga problemas que tenga que estar pensando, que tengo que lavar, que tengo que coser, que tengo cocinar porque eso le pasa a uno, porque uno está haciendo algo y está pensando que le falta otra cosa que hacer, entonces recién voy a hacer. Pero me decía mi hija usted va a estar vieja entonces, no importa porque siempre va a haber alguien que le enseñé a uno, por eso cuando llegué aquí el año pasado me junté con la Ignacia, la otra que viene mañana, y me dijo sabí Francisca fíjate que ahi en la escuela van a hacer clases a las viejas, hablaba así, van a hacerle clases a las viejas vamos.

\subsubsection{Facilitación por figura docente}

Existe unanimidad por parte de las participantes de este estudio en reconocer que las profesoras de sus respectivos planes facilitaron condiciones para asegurar aprendizajes. Además, el trabajo de la profesora también es percibido como facilitador de la motivación.

Excelente profesora, con paciencia única porque todas teníamos su genio, sobre todo la que está hablando en estos momentos, era muy apurona con la profesora. Excelente profesora, no la cambio para nada, a pesar de todo éramos como un grupo solo, éramos una sola profesora y una sola alumna, éramos todos unidos, fue bonito.

\subsubsection{Adaptación del plan al adulto mayor}

Una de las ventajas que las mujeres mayores adjudican al plan refiere a que perciben que se adapta a sus características, particularmente a su condición de adulto mayor. En este sentido, rescatan el ritmo y las actividades de enseñanza ofrecidas.

Los ayudaba mucho, por último, con pelotitas, con manzanitas y ahí uno iba entendiendo o sea yo creo que lo que yo sé, lo poco y nada que sé, yo lo aprendí ahí... La paciencia, lo que nosotras como personas mayores necesitamos.

\subsubsection{Adquisición de mayor empoderamiento}

La participación en el plan otorga variados beneficios de acuerdo a la perspectiva de las mujeres mayores. No obstante, la consecuencia que más aprecian es la obtención 
personal de mayor seguridad, confianza y percepción de poder. Esta percepción de mayor empoderamiento se reflejaba tanto en su participación en el plan como en otros escenarios sociales.

\begin{abstract}
Acá me sirvió mucho, me ha servido a tener confianza para llegar a estudiar acá de nuevo porque uno siempre siente vergüenza, dice "oh, cómo, qué se yo". $\Upsilon$ aquí Contigo Aprendo, con la señorita, me sirvió mucho. Lo que aprendí y lo que he aprendido en mi vida.

Como que uno toma más personalidad para enfrentarse a la vida y tratar de pronunciar bien las palabras. De leer y entender lo que uno aprendió. Porque a uno a veces leía, juntaba las letras, pero no entendía. Entonces uno aprendía a conversar con más calma. Eso ha dado más seguridad.
\end{abstract}

\title{
3.4.5. Satisfacción por obtención de aprendizajes
}

Desde el discurso de las mujeres mayores, es posible identificar un significativo nivel de satisfacción por los aprendizajes experimentados producto de su participación en el plan de alfabetización. Además, le atribuyen un alto valor dado que tales aprendizajes permiten reconocimiento social.

Un orgullo muy grande, un orgullo. Porque cualquiera no me va a decir oye a ver junta las letras. No, yo las leo.

\subsubsection{Satisfacción por relaciones de apoyo}

Las participantes señalan que fueron gratificantes las relaciones interpersonales que establecieron con sus compañeras en el plan. Estas relaciones les permitían obtener otra fuente de apoyo adicional a la ofrecida por la profesora. Inclusive, muchas se percibían como un apoyo constante para facilitar el aprendizaje de las demás.

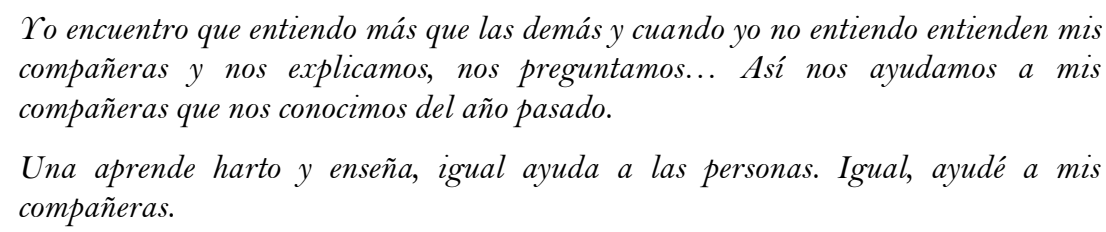

\subsection{Esquema y argumento de la historia de la identidad narrativa}

La figura 1 contiene las categorías construidas en la codificación abierta, las cuales se encuentran relacionadas mediante fechas que fueron delineadas en la codificación axial. Adicionalmente, se muestra en la misma figura la segmentación estructurada en cuatro etapas: infancia, adultez, vejez y plan. Estas etapas con sus categorías fueron articuladas durante la codificación de proceso. Cabe señalar que la etapa plan es en rigor una subetapa de la vejez.

A continuación, se describirá el esquema que se muestra en la figura 1 a partir de la técnica del argumento de la historia. Es decir, se narrará integrada y resumidamente la historia de las participantes a partir de la secuencia de categorías construidas y se analizarán en base al derecho a la educación. En primer lugar, durante la infancia de las mujeres se presentan condiciones familiares con limitaciones socioeconómicas y con una negativa relación con alguno de sus padres o cuidadores. Estas condiciones les imponen dedicarse a labores de cuidado, domésticas y/o del campo, las cuales son rechazadas por las mujeres y generan la intención de escapar. Junto con un disminuido nivel de bienestar, las mujeres en su niñez experimentan una vulneración del derecho a la educación producto de las determinaciones de sus cuidadores y/o de la situación de pobreza que atravesaron, restringiendo su accionar a tareas fuera del ámbito escolar. 
Dadas las condiciones de su infancia, las mujeres generan una concepción de sí mismas desde el género femenino, posicionando como central en su identidad la orientación al servicio de los demás con labores de cuidado y domésticas. Esta autoconcepción hace que la intención de escapar se traduzca en embarazo y casamiento, marcando la entrada a la adultez. No obstante, el matrimonio se experimenta negativamente, dado que el esposo niega la posibilidad de incorporarse a procesos educativos. Esta vulneración tiene consecuencias negativas, especialmente la vergüenza por no disponer de aprendizajes como la lectoescritura.

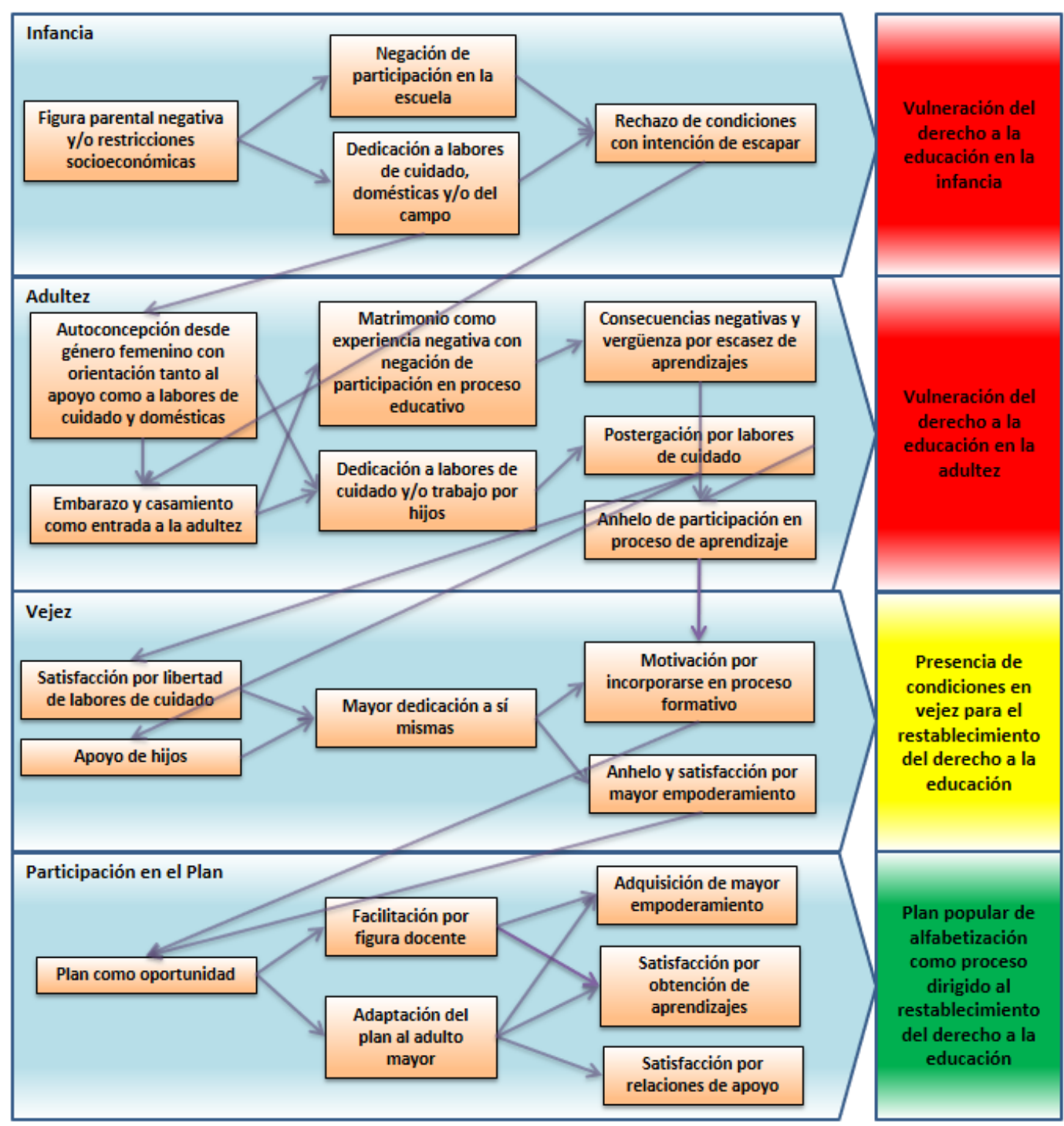

Figura 1. Esquema de la identidad narrativa de las mujeres mayores que participaron en el plan Contigo Aprendo

Fuente: Elaboración propia.

Como el tiempo en la adultez es utilizado fundamentalmente para labores de cuidado y/o para trabajar por los hijos, se postergan los propios intereses. La vergüenza y la postergación vivenciada generan que en tal periodo se mantenga el anhelo de participar en procesos de aprendizaje. El derecho a la educación de las mujeres en esta fase se encontraría en una intensa vulneración. Aunque este proceso está influido también por determinaciones de terceros, en este caso de la pareja, juega un papel sustancial la 
autoconcepción basada en el servicio que mantiene la postergación de instancias educativas. Esta vulneración tensa la dimensión afectiva dado que se presenta un interés por activar el goce del derecho a la educación, interés potenciado por sentimientos de descrédito.

Por su parte, la entrada a la vejez se vive con satisfacción al contar tanto con mayor libertad de labores de cuidado como con el apoyo de sus hijos. Estas condiciones les permiten dedicarse a sí mismas y reactivar su motivación para incorporarse en un proceso de aprendizaje. Además, sienten que anhelan y disfrutan de un mayor empoderamiento. Conjuntamente, estos elementos generan condiciones favorables para restablecer el derecho a la educación. Si bien algunas de estas condiciones son externas, como el soporte brindado por los hijos, la mayor parte alude a un cambio actitudinal respecto a la dedicación personal, la cual se focaliza a sí mismas. Puesto que esta nueva actitud es acompaña tanto con una mayor percepción de control personal como con una motivación legítima por insertarse en procesos educativos, se posibilita el disfrute del derecho a la educación como un derecho ejercido por sí mismas y no como simplemente algo otorgado y/o restringido por otros.

El estado motivacional de las mujeres mayores hace que vean el plan de alfabetización como una valiosa oportunidad. Una vez incorporadas en el plan, reconocen que se adapta a su condición de adulto mayor y experimentan la facilitación que realiza el docente. Estas características permiten que refuercen su empoderamiento y seguridad, obtengan aprendizajes y disfruten de relaciones interpersonales de apoyo. Es precisamente en el contexto de este plan popular donde el goce del derecho a la educación de las mujeres mayores se materializa, respondiendo a la intención acentuada en la etapa de la vejez por desarrollar aprendizajes en un espacio educativo. Es importante destacar que el disfrute del derecho a la educación se concretiza a partir de las características flexibles del plan de alfabetización, pero también con la comunidad social que allí se origina. En la esfera afectiva se presenta una significativa gratificación derivada del ejercicio del derecho a la educación, al tomar consciencia de su propio desarrollo y de la comunidad constituida.

\section{Discusión y conclusiones}

En la identidad narrativa de las mujeres mayores que fue presentada en la sección de resultados se muestran aspectos claves para comprender cómo se originó y se mantuvo a lo largo de la infancia y la adultez la vulneración del derecho a la educación. Como es posible constatar, la vulneración estuvo basada íntimamente en la adherencia a un sistema de significados que avalaba distintos estereotipos de género. De esta forma, las narraciones de las participantes pueden comprenderse al tomar en cuenta que los sistemas de significados van constituyendo la mente y condiciona las interpretaciones que hacen de sí mismos (Bruner, 1990).

Desde su entorno familiar próximo, figuran personas que ejercen un control social para mantenerlas en el espacio cultural privado con objeto de que desempeñen roles sujetos al estereotipo de género femenino: los padres y el esposo. Este control social estaba cimentado en una asimetría de poder respaldada en el sistema valórico sin considerar los intereses personales de las mujeres y, por tanto, puede considerarse como una forma de opresión. Estas restricciones son también identificadas por Valdés (1987) y, por tanto, son típicas en contextos familiares con presencia de estos estereotipos. 
Por otro lado, es posible sostener que la práctica comunitaria basada en estereotipos de género que demarcó la vida cotidiana de las mujeres durante la infancia y la adultez, fue asimilada en su identidad. Este proceso se puede comprender considerando que las relaciones interpersonales recurrentes estaban limitadas a un escenario social específico: el hogar. En efecto, la formación de la persona es un resultado de las relaciones de mutua constitución entre individuos (Wenger, 2002). Esta esfera restringida de participación hacía que los sistemas disponibles de significados ofrecieran perspectivas restrictivas para interpretarse a sí mismas. En esta línea, las mujeres significan la disponibilidad de tiempo libre y el apoyo ofrecido por los hijos como una consecuencia merecida y, por ende, se validan principalmente desde el rol de madre.

Asimismo, es importante destacar que el interés por ejercer el derecho a la educación estaba presente desde antes del ingreso al plan popular de alfabetización y no es generada por éste. Dicha intención puede solventarse, desde la perspectiva de las mujeres, una vez que "cumplan" con sus responsabilidades ligadas a su género. Por consiguiente, se atribuye legitimidad a la decisión de incorporarse en la etapa de la vejez bajo la aprobación del sistema cultural aludido. Además, este interés se ve reforzado cuando las mujeres se trasladan al mundo público en donde los sistemas simbólicos internalizados se superponen con otros. Al advertirlos, se produce a nivel afectivo una fuerte vergüenza al tomar conciencia de no contar con habilidades relacionadas con la alfabetización que se esperan desde estos otros sistemas culturales.

De este modo, el Plan Contigo Aprendo se convierte en la oportunidad de participar en un espacio que les permite aprender a leer y escribir y, con ello, concretizar un anhelo que estuvo presente desde la niñez y que por las condiciones socioculturales opresoras no pudieron realizar. En palabras de Freire (1985), este anhelo correspondería a las ansias que poseen los personas oprimidas para luchar por la libertad y recuperar su humanidad. Pero esta lucha requiere de la unión con otros oprimidos, ya que las personas se liberan en comunión. En concordancia con lo anterior, las participantes destacan las relaciones de apoyo que construyeron con sus compañeras y monitora, siendo estas esenciales para la construcción de aprendizajes y reforzar su seguridad. Según Arrufat (2004), las relaciones de apoyo entre mujeres son la base para que puedan avanzar todas juntas, tanto académicamente como en otras esferas, hacia la superación de las desigualdades de todas las mujeres.

Por su parte, este plan está enfocado a los adultos y, por ende, tiene características que sus participantes destacan y que permiten su adherencia durante todo el periodo de trabajo. Lo anterior es sumamente relevante si se considera que las participantes deben enfrentar sentimientos de vergüenza que han anidado a lo largo de su vida, por lo cual, contar con un plan que, desde su perspectiva, se adecua a sus necesidades y considera sus experiencias permite que se comprometan con su proceso educativo. Lo anterior, es concordante con lo planteado por Esquivel (1999), ya que esta valorización de experiencias y saberes previos hacen que el enfoque de la educación popular sea particularmente útil para el trabajo con los adultos mayores.

Es así como la participación en el plan Contigo Aprendo ha permitido que las mujeres mayores desarrollen las habilidades básicas de alfabetización, transformándose así este espacio en una plataforma para restituir el derecho a la educación, ya que la alfabetización es inherente a este derecho y es la base sobre la cual se construye el aprendizaje (UNESCO, 2009). De este modo, a medida que las mujeres mayores van 
desarrollando las habilidades de alfabetización, comparten experiencias con sus compañeras y monitora, sintiéndose más seguras de sí mismas. Este aumento en la adquisición de confianza gracias a la alfabetización, al reconocimiento de sus experiencias y saberes y al diálogo constante en relación a estos, produce un mayor empoderamiento de las mujeres, el cual les permite sentirse capaces de liderar las distintas esferas de su vida (UNESCO, 2017). Este proceso se ha producido a lo largo de su experiencia en el plan, por ende y en pal abras de Freire (2004), se ha producido una creación y re-creación personal a partir de las vivencias en ese espacio, lo cual permitió que actualmente las participantes se consideren más seguras y que se sientan satisfechas con sus aprendizajes y con las relaciones que establecieron en el plan.

Se identifican dos importantes conclusiones en esta investigación. En primer lugar, se destaca el papel de sistemas culturales que respaldan estereotipos de género para impactar el ámbito familiar, la historia de vida y la identidad de las mujeres, restringiendo su derecho a la educación. En segundo término, se identifica como una práctica social transformadora el plan popular de alfabetización para que las mujeres puedan restituir su derecho a la educación.

Una de las limitaciones de este estudio se relaciona con la metodología empleada, tomando en cuenta que se realizó una reconstrucción del proceso de participación en el plan popular de alfabetización a partir del pasado de las mujeres mayores. Por tanto, no se estudia las reflexiones de las mujeres mayores en el curso de su participación presente. Otra limitación identificada refiere a que existen diversas instancias populares de alfabetización y este estudio indagó en un plan específico.

En el marco de estas limitaciones, futuras investigación podrían adquirir un enfoque longitudinal bajo metodologías etnográficas y/o de investigación acción para capturar las experiencias de las mujeres mayores en el presente. Adicionalmente, se considera pertinente incluir con mayor variedad modalidades populares de trabajo a fin de realizar un análisis comparativo de diversas estrategias de la educación popular en materia de alfabetización de adultos mayores.

\section{Referencias}

Arciero, G. (2005). Estudios y diálogos sobre identidad personal: Reflexiones sobre la experiencia humana. Buenos Aires: Amorrortu.

Bolívar, A. (2002). "¿De nobis ipsis silemus?": Epistemología de la investigación biográficonarrativa en educación. Revista Electrónica de Investigación Educativa, 4(1), art. 4.

Bruner, J. (1990). Actos de significado. Más allá de la revolución cognitiva. Madrid: Alianza.

Bruner, J. (2004). Realidad mental y mundos posibles. Los actos de imaginación que dan sentido a la experiencia. Barcelona: Gedisa.

Cannobbio, L. y Jeri, T. (2008). Estadísticas sobre las personas adultas mayores: Un análisis de género. Santiago de Chile: SENAMA.

Comboni, S. y Juárez, J. (2005). Educación de adultos en América Latina: Perspectivas en los albores del siglo XXI. Revista Interamericana de Educación de Adultos, 27(1), 21-69.

Comunidad Mujer. (2016a). Informe GES: Género, educación y trabajo. Santiago de Chile: Comunidad Mujer. 
Comunidad Mujer. (2016b). Mujer y trabajo: Los retos que plantea la feminización de la vejez en Chile. Santiago de Chile: Comunidad Mujer.

Didier, M. (1990). Participación de los sectores pobres en programas de desarrollo local. Santiago de Chile: UNICEF.

Eltit, D. (1994). Crónica del sufragio femenino en Chile. Santiago de Chile: SERMAN.

Escobar, D., Berlien, K. y Ostoic, D. (2016). Informe final de evaluación: Programa educación para personas jóvenes y adultas. Santiago de Chile: Ministerio de Educación.

Esquivel, F. (1999). Educación popular: Su aprovechamiento con el adulto(a) mayor, un acercamiento al teatro popular. Revista Costarricense de Trabajo Social, 9, 75- 83.

Flick, U. (2007). Introducción a la investigación cualitativa. Madrid: Morata.

Freire, P. (1985). Pedagogía del oprimido. Buenos Aires: Siglo XXI.

Freire, P. (2004). La educación como práctica de libertad. Buenos Aires: Siglo XXI.

García, N. (2007). La educación con personas mayores en una sociedad que envejece. Horizontes Educacionales, 12(2), 51-62.

Hermanns, H. (1995). Narratives interview. En U. Flick, E. Kardorff, H. Keupp, L. Rosenstiel y S. Wolff (Eds.), Handbuch qualitative sozialforschung (pp. 182-185). Munich: Psychologie Verlags Union.

Hernández, G. (2009). Impactos de un taller de alfabetización de adultos en la vida cotidiana de sus participantes (Tesis de Magíster). Universidad de Chile.

Lampert-Grassi, M. (2014). Sistematización de políticas sociales dirigidas al adulto mayor. Santiago de Chile: Biblioteca del Congreso Nacional de Chile.

Ministerio de Desarrollo Social. (2015). Encuesta de caracterización socioeconómica nacional. Santiago de Chile: Ministerio de Desarrollo Social.

Ministerio de Educación. (2017). ¿Qué es el plan de alfabetización "Contigo aprendo"? Recuperado de https://epja.mineduc.cl/plan-de-alfabetizacion/estudiantes/plan-alfabetizacion-contigoaprendo

Mogollón, E. (2012). Una perspectiva integral del adulto mayor en el contexto de la educación. Revista Interamericana de Educación de Adultos, 34(1), 57-74.

Ricoeur, P. (1995). Tiempo y narración I: Configuración del tiempo en el relato histórico. Ciudad de México: Siglo XXI.

Strauss, A. y Corbin, J. (2002). Bases de la investigación cualitativa. Medellín: Editorial de la Universidad de Antioquia.

Susinos, T. y Parrilla, Á. (2008). Dar la voz en la investigación inclusiva. debates sobre inclusión y exclusión desde un enfoque biográfico-narrativo. REICE. Revista Iberoamericana sobre Calidad, Eficiencia y Cambio en Educación, 6(2), 157-171.

Tezanos, J. F. (2001). Tendencias en desigualdad y exclusión social. Madrid: Sistema.

Torres, A. (2007). Paulo Freire y la educación popular. Revista Educación de Adultos y Desarrollo, 69(1), 45-77.

UNESCO. (2009). Aprovechar el poder y el potencial del aprendizaje y la educación de adultos para un futuro viable. Recuperado de http://www.uil.unesco.org/fileadmin/multimedia/uil/confintea/pdf/National_Reports/L atin\%20America\%20-\%20Caribbean/Colombia.pdf 
UNESCO. (2010). Relatório Global sobre a Aprendizagem e a Educação de Adultos. Recuperado de http://unesdoc.unesco.org/images/o018/001886/188644por.pdf

UNESCO. (2017). Educación al servicio de los pueblos y el planeta: creación de futuros sostenibles para todos, informe de seguimiento de la educación en el mundo. Recuperado de http://unesdoc.unesco.org/images/o024/002485/248526S.pdf

Valdés, T. (1987). Ser mujer en sectores populares. En H. Pozo (Ed.), Espacio y poder, los pobladores (pp. 203-258). Santiago de Chile: FLACSO.

Verba, S., Nie, N. H. y Kim, J. O. (1978). Participation and political equality: A seven-nation comparison. Cambridge: Cambridge University Press.

Wenger, E. (2002). Comunidades de Práctica: aprendizaje, significado e identidad. Madrid: Paidós.

World Health Organization. (2016). World Health Stadistics: Monitoring health for the sustentable development goals. Recuperado de http://www.who.int/gho/publications/world_health_statistics/2016/en/

\section{Breve CV de los autores}

\section{Karina Moreno Díaz}

Psicóloga titulada de la Universidad de Santiago de Chile y Magíster en Psicología Educacional de la Universidad de Chile. Con experiencia en las áreas de Convivencia e Integración Escolar en Centros Educativos y en la coordinación y gestión técnica de Proyectos de Investigación en el ámbito educativo y desarrollo infantil. Ha realizado investigaciones en temáticas relacionadas con la inclusión educativa en situaciones de vulnerabilidad. Actualmente es la Encargada Fundacional de Orientación y Familia de la Fundación Belén Educa. ORCID ID: O000-0003-0312-735X. Email: karina.moreno@usach.cl

\section{Rodolfo Soto González}

Psicólogo titulado de la Universidad de Santiago de Chile y Magíster en Psicología Educacional de la Universidad de Chile. Con experiencia en docencia de nivel superior e intervenciones en centros educativos en ámbitos como inclusión, convivencia y aprendizaje. Ha realizado investigaciones en temáticas relacionadas con la educación inclusiva y segregación escolar. Actualmente es parte del cuerpo docente del Centro de Formación Técnica ENAC. ORCID ID: 0000-0003-3061-6036. Email: rodolfo.soto@usach.cl 